\title{
Growth and Characterization of an Organic Crystal and DFT Studies of 2-amino 5-methyl Pyridinium Salicylate
}

\author{
D PRABHA ${ }^{1}$, P HARISH$^{1}$, B BABU $^{2}$ and V SATHYA NARAYANA MOORTHI ${ }^{\text {* }}$ \\ 'Department of Physics, PSG College of Arts and Science, Coimbatore 641 014, India. \\ 2Department of Physics, Sri Ramakrishna Mission Vidyalaya College of Arts and Science, \\ Coimbatore- 641020, India \\ *Corresponding author Email: sathyanarayanamoorthi@yahoo.co.in
}

http://dx.doi.org/10.13005/ojc/320420

(Received: June 29, 2016; Accepted: July 30, 2016)

\begin{abstract}
Single crystals of 2-amino 5-methyl pyridinium salicylate (2A5MPSA) were grown by slow evaporation of a solvent ethanol (1:1) containing equimolar quantities of 2-amino 5-methyl pyridine and salicylic acid. Powder XRD study reveals the crystalline nature of the grown sample. FTIR study was carried out to identify the functional groups present in 2A5MPSA. Thermal behavior and stability of 2A5MPSA were investigated by thermogravimetric (TG) and differential thermal analysis (DTA). Transparency of the title crystal in UV-Vis-NIR region was analyzed. Study the electronic properties of 2A5MPSA using the B3LYP functional with 6-311G basis set. Mulliken's charges have been obtained using the DFT calculation method. The first order hyperpolarizability and the HOMO-LUMO energies, hardness, softness and ionization potential have been calculated.
\end{abstract}

Keywords: Crystal growth, Characterization, Density functional calculations, NLO calculations, HOMO-LUMO, Natural bond orbitals.

\section{INTRODUCTION}

Nonlinear optical materials are attracting a great deal of attention, because of their use in photonic devices, sensors and laser spectroscopy ${ }^{1-5}$. Many investigations are being carried out to synthesize new organic materials with large secondorder optical nonlinearities in order to satisfy dayto-day technological requirements ${ }^{6-7}$. The nonlinear (NLO) properties of materials can be used to reduce significantly the transmittance of a device for high inputs. Such a device is called optical limiters. The optical limiting materials have applications in the protection of optical sensors and human eyes from intense laser light. Many new organic crystals have been found based on the predictive molecular engineering approach and have been shown to have potential applications in non-linear optics and these materials find wide application in telecommunication, frequency mixing, optical parametric oscillation, optical bistability, optical image processing and under water communication etc. In recent years, research 
on co-crystals or organic salts has been expanded rapidly because of their potential applications in the field of material science, molecular biology, and pharmaceutical science.

The advantage of the organic materials is that they offer a high degree of synthetic flexibility to tailor their optical properties through structural modification and exhibit very high laser damage threshold. Organic crystals show large nonlinear optical properties. Organic materials have 10-100 times larger SHG co-efficiencies than inorganic materials, but they create crystal processing difficulties due to their softness and fragility. A major task for organic crystals for practical applications is to simultaneously achieve the desired arrangement of the constituting molecules in the crystalline state and optimal crystal characteristics with suitable morphology, habit, and dimension. The increasing demand for organic materials for technological applications, which include optical frequency doublers, ultrafast modulators, amplifiers, and switches. Well designed organic nonlinear optical active crystals show generally higher and faster nonlinearities are being conducted synthesize new organic materials with large second-order optical nonlinearties in order to satisfy day to day technological requirements. Pyridine has been commonly used as molecular building blocks of nonlinear optical materials as pyridine ring acts as a cationic bonding site, the nitrogen acts as proton acceptor these by becoming a strong organic base. Recently pyridine and their derivatives have been the subject of intensive studies for the potential role in the image processing and optical communication.

\section{MATERIALS AND METHODS}

\section{Synthesis and crystal growth}

Commercially available 2-amino 5-methylpyridine (Sigma 99\%) and Salicylic acid (Himedia 99\%) both reactants were taken in equimolar ratio and dissolved separately using ethanol and stirred well for about $20 \mathrm{~min}$. Then the solutions were mixed together and stirred for about $1 \mathrm{hr}$ using magnetic stirrer. The saturated solution was filtered twice using Whatmann filter paper and transferred to $250 \mathrm{ml}$ beaker. To control the solvent evaporation rate, top of the beaker was covered with a thin plastic sheet and kept at the undisturbed place for slow evaporation. After 10 days good quality crystals were acquired from the mother solution. The as crystal was depicted in Fig1.

\section{Characterization Techniques}

FTIR spectrum of 2-amino 5-methyl pyridinium salicylate (2A5MPSA) crystal was recorded using Perkin Elmer spectrometer in the range of $4000-400 \mathrm{~cm}^{-1}$ by employing $\mathrm{KBr}$ pellet method. In order to study linear optical characteristics, grown crystals of 2A5MPSA were subjected to UV-Vis-NIR spectral analysis in the wavelength range from 200 to $1200 \mathrm{~nm}$, with the help of Varian Cary UV-Vis-NIR spectrophotometer. The thermal properties of 2A5MPSA compound were analyzed by thermogravimetric and differential thermal (TGA/ DTA) analysis using instrument SDT Q 600. The thermal analyzer is done in the temperature range $26-600^{\circ} \mathrm{C}$ with a heating rate of $10^{\circ} \mathrm{C}$ per minute in a nitrogen atmosphere. Crystals were finally crushed and subjected to powder XRD analysis using Bruker AXS D8 Advance powder diffractometer with $\mathrm{Cu}$, wavelength $1.5406 \mathrm{~A}^{\circ}$ were used. The sample was scanned in the reflection mode in the $2 r$ range 10 $90^{\circ}$. The DFT calculations were performed using the GAUSSIAN 09 program package with most popular DFT model B3LYP (Becke3-Lee-Yang-Parr) at the basis set $6-311 \mathrm{G}$ and $6-311++\mathrm{G}$.

\section{RESULTS AND DISCUSSION}

\section{FTIR Spectral studies}

The recorded spectrum is shown in Fig 2. The $\mathrm{N}-\mathrm{H}$ stretching vibration of primary amine occurred at $3289 \mathrm{~cm}^{-1}$. A peak at $3214 \mathrm{~cm}^{-1}$ is due to stretching vibration of the secondary amine of $\mathrm{N}-\mathrm{H}^{+}$. The aromatic $\mathrm{C}-\mathrm{H}$ stretching vibration was observed at $2931 \mathrm{~cm}^{-1}$. The aromatic $\mathrm{C}=\mathrm{C}$ and $\mathrm{C}=\mathrm{N}$ stretching vibrations occurred at 1659 and $1611 \mathrm{~cm}^{-1}$ respectively. The aromatic $\mathrm{C}=\mathrm{H}$ plane bending mode of vibration appears at $1147 \mathrm{~cm}^{-1}$. The $\mathrm{C}-\mathrm{H}$ in-plane and out of plane bending vibrations are observed at 1021 and $860 \mathrm{~cm}^{-1}$. The $\mathrm{N}-\mathrm{H}$ out of plane bending vibration occurred at $670 \mathrm{~cm}^{-1}$.

\section{UV-Vis-NIR spectrum}

An NLO material needs to be transparent over a wide wavelength range of its practical applications and its lower cut off is to be between 200 $\mathrm{nm}$ to $400 \mathrm{~nm}$. The recorded spectrum is as shown 
in Fig3. The cut -off wavelength is the absence of absorption in the entire visible region.

\section{Thermal analysis}

The sample weighting $12.445 \mathrm{mg}$ was analyzed and the TG curve depicted Fig 4. The DTA curve indicates the same changes as shown in TG curve. The DTA curve indicates the same changes shown in TG curve. From the TG curve, it is evident that the material stable up to $175^{\circ} \mathrm{C}$ and moisture free. TG curve showed two stage mass loss patterns. When the material was heated 26 to $600^{\circ} \mathrm{C}$. The first mass loss occurred between the temperature $170^{\circ} \mathrm{C}$ and $200^{\circ} \mathrm{C}$. The DTA trace of 2-amino 5-methyl pyridinium salicylic acid is shown in Fig4. There is an endothermic peak at about $169.65^{\circ} \mathrm{C}$ which may be the melting point of the material and there is no corresponding weight loss in TGA trace. This is followed by a sharp endothermic peak at $231.10^{\circ} \mathrm{C}$ is due to the decomposition of major functional groups. From the above analysis, the material is thermally stable up to $170^{\circ} \mathrm{C}$ and it starts to decompose at $231.10^{\circ} \mathrm{C}$.

\section{Powder XRD}

The recorded spectrum is shown in Fig5. Sharp and well defined Bragg's peaks confirm the crystalline nature of the crystal. The maximum

Table 1: Theoretical electric dipole moment and hyperpolarizability values of 2-amino 5-bromo pyridinium salicylate

\begin{tabular}{lcccc}
\hline $\begin{array}{l}\text { Dipole } \\
\text { (Debye) }\end{array}$ & \multicolumn{2}{c}{$\begin{array}{c}\text { B3LYP/6-311G } \\
\text { Polarizability }\end{array}$} & $\begin{array}{c}\text { B3LYP/ 6-311G } \\
\text { Hyperpolarizability }\end{array}$ \\
\hline$\mu_{\mathrm{x}}-1.0326$ & $\alpha_{\mathrm{xx}}$ & 4.4527 & $\beta_{\mathrm{xxx}}$ & 3.2157 \\
$\mu_{\mathrm{y}} 1.5559$ & $\alpha_{\mathrm{yy}}$ & -2.1038 & $\beta_{\mathrm{XXY}}$ & 7.5201 \\
$\mu_{\mathrm{z}} 3.6082$ & $\alpha_{\mathrm{zz}}$ & 2.0335 & $\beta_{\mathrm{XYY}}$ & -2.7098 \\
& $\alpha_{\mathrm{xy}}$ & 1.5722 & $\beta_{\mathrm{YYY}}$ & 4.3797 \\
& $\alpha_{\mathrm{xz}}$ & 1.9404 & $\beta_{\mathrm{Xxz}}$ & -1.2782 \\
& $\alpha_{\mathrm{yz}}$ & 1.1078 & $\beta_{\mathrm{XYZ}}$ & 6.3858 \\
& $\alpha_{\mathrm{Total}}$ & 1.4608 & $\beta_{\mathrm{yyz}}$ & 1.6445 \\
& $(\mathrm{e} . \mathrm{s.u})$ & 0.2164 & $\beta_{\mathrm{xzz}}$ & 1.2806 \\
& & & $\beta_{\mathrm{yzz}}$ & 2.1864 \\
& & & $\beta_{\mathrm{zzz}}$ & 3.6564 \\
& & & $\beta_{\mathrm{Total}(\mathrm{a.u})}$ & 14.669 \\
& & & $(\mathrm{e} . \mathrm{s} . \mathrm{u})$ & 126.7368
\end{tabular}

intensity of 15754 counts was observed for the crystal.

\section{Quantum chemical studies NLO calculations}

Recently, a compound having nonlinear optical (NLO) properties have got the appreciable attention of researchers because of their wide applications in optoelectronic devices of telecommunications, information storage, optical switching and signal processing. Molecules containing donor-acceptor groups along with pi-

Table 2: Mullilken atomic charge of 2A5MPSA

\begin{tabular}{lc}
\hline Atoms & B SLYP/6311++G \\
\hline $\mathrm{C}_{1}$ & -0.837959 \\
$\mathrm{C}_{2}$ & 0.442754 \\
$\mathrm{C}_{3}$ & -0.475874 \\
$\mathrm{C}_{4}$ & 1.002836 \\
$\mathrm{C}_{5}$ & 0.149510 \\
$\mathrm{~N}_{6}$ & -1.126175 \\
$\mathrm{C}_{7}$ & -1.982196 \\
$\mathrm{~N}_{8}$ & -0.444696 \\
$\mathrm{H}_{9}$ & 0.338389 \\
$\mathrm{H}_{10}$ & 0.274088 \\
$\mathrm{H}_{11}$ & 0.449090 \\
$\mathrm{H}_{12}$ & 0.852322 \\
$\mathrm{H}_{13}$ & 0.238377 \\
$\mathrm{H}_{14}$ & 0.305092 \\
$\mathrm{H}_{15}$ & 0.238075 \\
$\mathrm{H}_{16}$ & 0.332124 \\
$\mathrm{H}_{17}$ & 0.422696 \\
$\mathrm{C}_{18}$ & -0.182000 \\
$\mathrm{C}_{19}$ & 1.306867 \\
$\mathrm{C}_{20}$ & -0.358406 \\
$\mathrm{O}_{21}$ & -0.586719 \\
$\mathrm{C}_{22}$ & -0.466204 \\
$\mathrm{C}_{23}$ & -0.627324 \\
$\mathrm{C}_{24}$ & -0.253302 \\
$\mathrm{C}_{25}$ & -0.751595 \\
$\mathrm{O}_{26}$ & 0.354839 \\
$\mathrm{O}_{27}$ & 0.386914 \\
$\mathrm{H}_{28}$ & \\
$\mathrm{H}_{29}$ & 0.301395 \\
$\mathrm{H}_{30}$ & \\
$\mathrm{H}_{31}$ & \\
\hline & \\
\hline & \\
\hline
\end{tabular}


electron conjugated system are considered as strong candidates for processing NLO properties.

In general, the physicochemical properties of the crystals are directly associated with their molecular inter and intramolecular interactions. The quantum chemical calculations were carried out to predict the nature of electric dipole moment, polarizability, chemical hardness, charge distribution, nucleophilic and electrophilic reactive sites of the 2A5MPSA molecule using density functional theory (DFT). The theoretical values of fundamental NLO parameters of 2A5MPSA obtained through the finitefield approach are listed in Table1. From Table 1, the polarizability (á) and the first order hyperpolarizability (â) of 2A5MPSA molecule were found to be 0.2164 esu and 126.7368 esu respectively. As urea is one of the prototypical molecules used in the study of NLO properties of molecular systems, it was used frequently as a threshold value for comparative

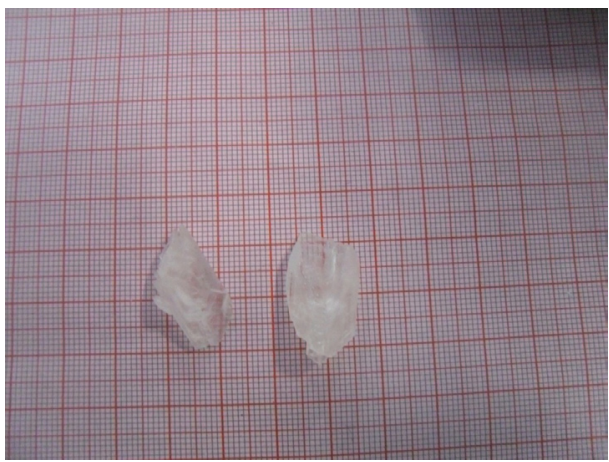

Fig. 1: Grown crystal of 2A5MPSA purpose. The obtained â of 2A5MPSA was about 145 times greater than that of urea $\left(\hat{\mathrm{a}}=0.8755 \times 10^{-30}\right.$ esu) ${ }^{9-10}$.

\section{Mulliken atomic charge}

Mulliken atomic charge calculation has an important role in the applications of quantum chemical calculations to molecular systems because of atomic charge effect, dipole moment, molecular polarizability, electronic structure and a lot of properties of molecular systems ${ }^{11}$. The calculated Mulliken charge values are listed in Table 2. From the table, we observed that the carbon atoms exhibit a substantial negative charge which is donor atoms. Hydrogen atoms exhibit a positive charge which is an acceptor atom. It is worthy to mention that $\mathrm{C}_{1}, \mathrm{C}_{3}$, $\mathrm{C}_{7}, \mathrm{C}_{18}, \mathrm{C}_{20}, \mathrm{C}_{22}$ and $\mathrm{O}_{26}$ atoms of 2-amino 5-methyl

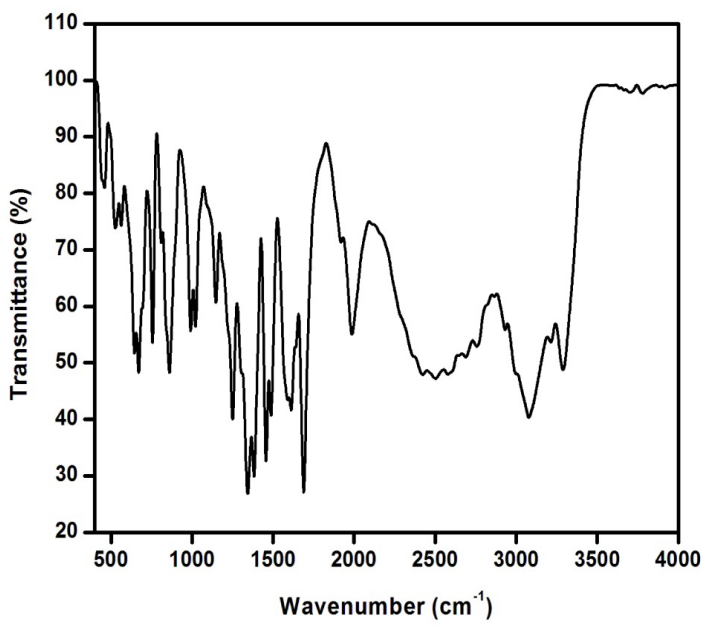

Fig. 2: FTIR spectrum of 2A5MPSA

Table 3: HOMO, LUMO, energy gaps and related molecular properties of 2A5MPSA crystal $(\mathrm{eV})$

\begin{tabular}{lc}
\hline Molecular properties & B3LYP/6-311++G \\
\hline $\mathrm{E}_{\text {HOMO }}(\mathrm{eV})$ & 3.2969 \\
$\mathrm{E}_{\mathrm{LUMO}}(\mathrm{eV})$ & -1.2808 \\
Energy gap $\left(\mathrm{E}_{\mathrm{HOMO}}-\mathrm{E}_{\mathrm{LUMO}}\right)$ & 2.0161 \\
lonization energy $(\mathrm{IE})=-\mathrm{E}_{\text {Hомо }}$ & 3.2969 \\
Electron affinity $(\mathrm{EA})=-\mathrm{E}_{\mathrm{LUMO}}$ & 1.2808 \\
Global hardness $(\mathrm{c})=1 / 2\left(\mathrm{E}_{\mathrm{HOMO}}-\mathrm{E}_{\mathrm{LUMO}}\right)$ & 1.0080 \\
Softness $(\mathrm{S})=1 / \eta$ & 0.9920 \\
Electronic chemical potential $(\mu)=1 / 2\left(\mathrm{E}_{\mathrm{HOMO}}+\mathrm{E}_{\mathrm{LUMO}}\right)$ & 2.2888 \\
Global electrophilicity $(\omega)=\mu^{2} / 2 \mathrm{ç}$ & -2.5986 \\
\hline
\end{tabular}


pyridinium salicylic acid exhibit a negative charge and $\mathrm{C}_{7}$ has a maximum negative charge value of above -1.9821 . The maximum positive atomic charges obtained for $\mathrm{C}_{2}, \mathrm{C}_{4}, \mathrm{C}_{5}$ and $\mathrm{C}_{19}$. The net positive charge of hydrogen atom suggests that the formation of intramolecular interactions of solid forms.

\section{Frontier molecular orbital}

B3LYP/6-311++G method has been employed to compute the chemical parameters, viz., molecular orbital (HOMO and LUMO) energies, energy gap $(\Delta E)$ electron affinity $(E A)$, ionization

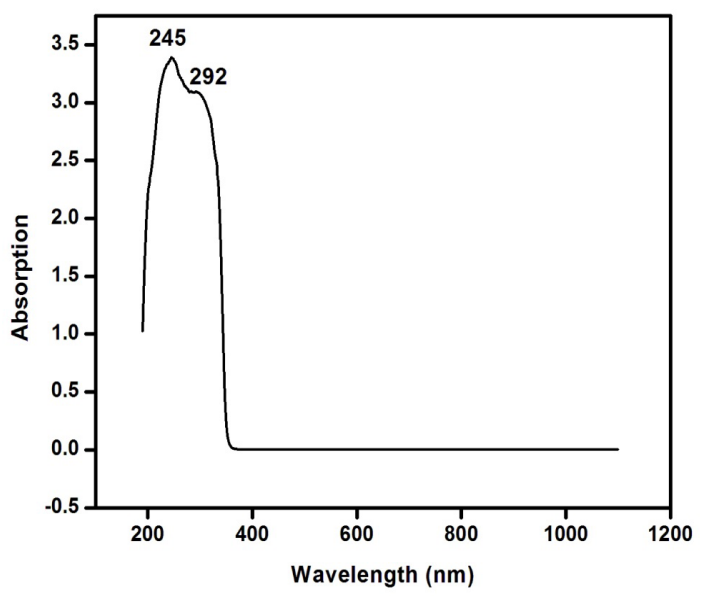

Fig. 3: Optical transmittance spectrum of 2A5MPSA potential (IP), hardness $(\chi)$, dipole moment $(\mu)$, softness (S), absolute electronegativity $(\chi)$ and electrophilicity index $(\omega)$ as shown in Table 3. The frontier orbital gap reflects in characterizing the chemical reactivity and kinetic stability of the molecule. The HOMO is the orbital that mainly act as an electron donor and the LUMO is the orbital that mainly acts as an electron acceptor. The energy gap HOMO (3.2969) to LUMO (-1.2808) of the molecule is about $2.0161 \mathrm{eV}^{12}$. The HOMO and LUMO energy explain the eventual charge transfer interactions taking place within the molecule. The electrophilicity is a significant parameter which is a measure of the molecule to accept the electrons. Nucleophilicity is an index of the molecule to donate the electrons. Thus, the electrophilicity and nucleophilicity of a molecule influence the electronic property of the molecule. The molecule with higher electrophilicity values will be a poor donor whereas the molecule having higher nucleophilicity will function as good donor. Thus, the reactivity of molecules and atoms are directly related to the ionization potential. But the softness and hardness of molecules give information about stability in addition to the reactivity.

\section{NBO Analysis}

The natural bond orbital analysis is an efficient method for studying intra-and intermolecular bonding and inter among bonds, and provides a convenient basis to probe charge transfer or conjugative interaction ${ }^{13}$. The NBO approach

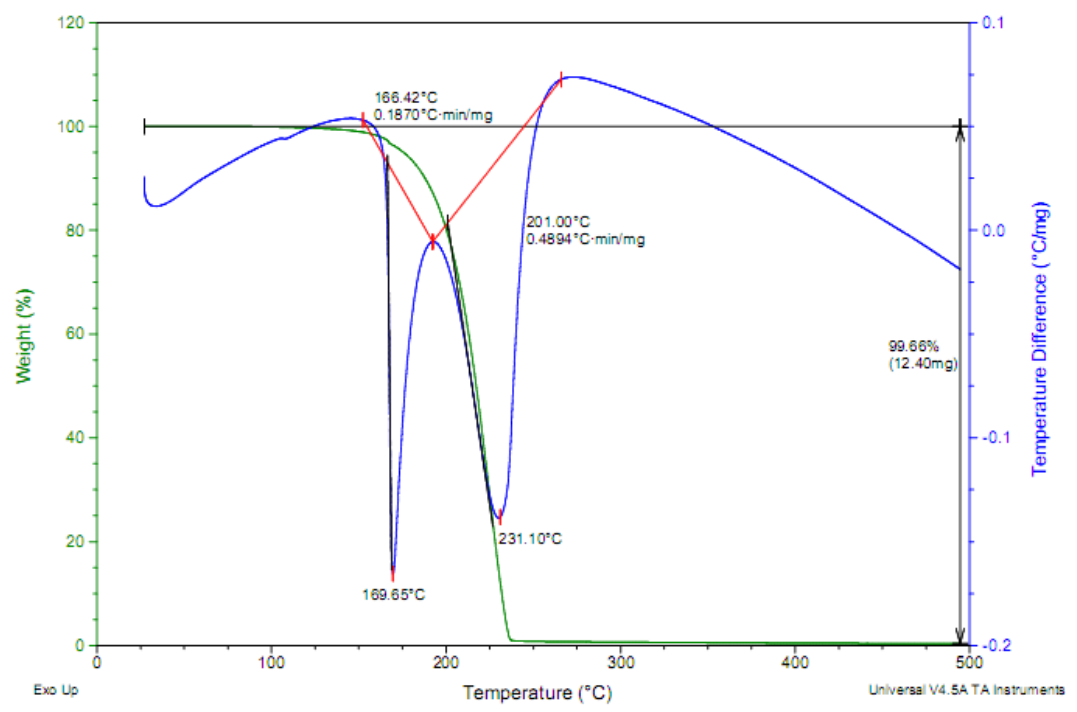

Fig. 4: TG/DTA spectrum of 2A5MPSA 
Table 4: Second-order perturbation theory analysis of Fock matrix in NBO basis for 2A5MPSA crystal

\begin{tabular}{|c|c|c|c|c|c|c|c|c|}
\hline Donar(i) & Type & ED/e & Acceptor(j) & Type & ED/e & $\begin{array}{c}\mathrm{E}(2) \\
\mathrm{kcal} / \mathrm{mol}\end{array}$ & $\begin{array}{c}E(j)-E(i) \\
\text { a.u. }\end{array}$ & $\begin{array}{c}F(i, j) \\
\text { a.u. }\end{array}$ \\
\hline \multirow[t]{3}{*}{$C_{1}-C_{2}$} & \multirow[t]{3}{*}{$\sigma$} & \multirow[t]{3}{*}{1.98} & $\mathrm{C}_{1}-\mathrm{N}_{8}$ & $\sigma^{*}$ & 0.01 & 2.15 & 1.52 & 0.051 \\
\hline & & & $\mathrm{C}_{2}-\mathrm{H}_{9}^{8}$ & $\sigma^{*}$ & 0.007 & 0.81 & 1.58 & 0.032 \\
\hline & & & $\mathrm{N}_{8}^{2}-\mathrm{H}_{16}$ & $\sigma^{*}$ & 0.01 & 1.18 & 1.53 & 0.038 \\
\hline \multirow[t]{3}{*}{$C_{1}-C_{2}$} & \multirow[t]{3}{*}{$\pi$} & & $\mathrm{C}_{3}-\mathrm{C}_{4}$ & $\pi^{*}$ & 0.24 & 15.53 & 0.62 & 0.089 \\
\hline & & & $\mathrm{C}_{5}-\mathrm{N}_{6}$ & $\pi^{*}$ & 0.73 & 26.01 & 0.30 & 0.094 \\
\hline & & & $\mathrm{N}_{8}-\mathrm{H}_{16}$ & $\sigma^{*}$ & 0.01 & 4.52 & 0.96 & 0.061 \\
\hline \multirow{7}{*}{$\mathrm{C}_{1}-\mathrm{N}_{6}$} & \multirow[t]{7}{*}{$\sigma$} & \multirow[t]{7}{*}{1.94} & $\mathrm{C}_{2}-\mathrm{C}_{3}$ & $\sigma^{*}$ & & 0.55 & 1.44 & 0.025 \\
\hline & & & $\mathrm{C}_{2}-\mathrm{H}_{9}$ & $\sigma^{*}$ & & 1.68 & 1.67 & 0.048 \\
\hline & & & $\mathrm{C}_{5}-\mathrm{N}_{6}$ & $\sigma^{*}$ & 0.03 & 5.67 & 1.58 & 0.085 \\
\hline & & & $\mathrm{C}_{5}-\mathrm{H}_{11}$ & $\sigma^{*}$ & & 3.02 & 1.68 & 0.064 \\
\hline & & & $\mathrm{N}_{6}-\mathrm{O}_{27}$ & $\sigma^{*}$ & 0.04 & 3.99 & 2.94 & 0.097 \\
\hline & & & $\mathrm{N}_{8}-\mathrm{H}_{17}$ & $\sigma^{*}$ & & 0.67 & 1.60 & 0.030 \\
\hline & & & $\mathrm{H}_{12}-\mathrm{O}_{27}$ & $\sigma^{*}$ & 0.06 & 15.30 & 3.61 & 0.210 \\
\hline \multirow[t]{9}{*}{$C_{1}-N_{8}$} & \multirow[t]{9}{*}{$\sigma$} & \multirow[t]{9}{*}{1.98} & $\mathrm{C}_{1}-\mathrm{C}_{2}$ & $\sigma^{*}$ & & 2.53 & 3.01 & 0.078 \\
\hline & & & $\mathrm{C}_{1}-\mathrm{N}_{6}$ & $\sigma^{*}$ & 0.08 & 0.59 & 2.75 & 0.037 \\
\hline & & & $\mathrm{C}_{1}-\mathrm{N}_{8}$ & $\sigma^{*}$ & & 0.65 & 2.83 & 0.038 \\
\hline & & & $\mathrm{C}_{2}-\mathrm{C}_{3}$ & $\sigma^{*}$ & & 244.07 & 2.66 & 0.720 \\
\hline & & & $\mathrm{C}_{4}-\mathrm{C}_{5}$ & $\sigma^{*}$ & & 16.49 & 2.99 & 0.199 \\
\hline & & & $\mathrm{C}_{5}-\mathrm{N}_{6}$ & $\sigma^{*}$ & & 4.23 & 2.80 & 0.098 \\
\hline & & & $\mathrm{C}_{5}-\mathrm{H}_{11}$ & $\sigma^{*}$ & & 5.77 & 2.90 & 0.115 \\
\hline & & & $\mathrm{N}_{6}-\mathrm{O}_{27}$ & $\sigma^{*}$ & 0.04 & 1.01 & 4.15 & 0.058 \\
\hline & & & $\mathrm{N}_{8}-\mathrm{H}_{17}$ & $\sigma^{*}$ & & 15.36 & 2.81 & 0.186 \\
\hline \multirow[t]{8}{*}{$C_{2}-C_{3}$} & \multirow[t]{8}{*}{$\sigma$} & \multirow[t]{8}{*}{1.97} & $C_{1}-C_{2}$ & $\sigma^{*}$ & & 1.87 & 1.68 & 0.050 \\
\hline & & & $\mathrm{C}_{1}-\mathrm{N}_{6}$ & $\sigma^{*}$ & 0.08 & 0.66 & 1.41 & 0.028 \\
\hline & & & $C_{1}-N_{8}$ & $\sigma^{*}$ & 0.01 & 4.47 & 1.49 & 0.073 \\
\hline & & & $\mathrm{C}_{2}-\mathrm{C}_{3}$ & $\sigma^{*}$ & & 2.00 & 1.32 & 0.046 \\
\hline & & & $\mathrm{C}_{2}-\mathrm{H}_{9}$ & $\sigma^{*}$ & 0.007 & 0.99 & 1.55 & 0.035 \\
\hline & & & $\mathrm{C}_{3}-\mathrm{C}_{4}$ & $\sigma^{*}$ & 0.02 & 8.32 & 2.00 & 0.115 \\
\hline & & & $\mathrm{C}_{3}-\mathrm{H}_{10}$ & $\sigma^{*}$ & & 1.20 & 1.54 & 0.038 \\
\hline & & & $C_{4}-C_{7}$ & $\sigma^{*}$ & 0.01 & 7.37 & 1.58 & 0.097 \\
\hline \multirow[t]{5}{*}{$\mathrm{C}_{2}-\mathrm{H}_{9}$} & \multirow[t]{5}{*}{$\sigma$} & \multirow[t]{5}{*}{1.98} & $C_{1}-C_{2}$ & $\sigma^{*}$ & & 1.07 & 1.48 & 0.036 \\
\hline & & & $\mathrm{C}_{1}-\mathrm{N}_{6}$ & $\sigma^{*}$ & 0.08 & 6.56 & 1.21 & 0.081 \\
\hline & & & $\mathrm{C}_{2}-\mathrm{C}_{3}$ & $\sigma^{*}$ & & 1.06 & 1.12 & 0.031 \\
\hline & & & $\mathrm{C}_{3}-\mathrm{C}_{4}$ & $\sigma^{*}$ & 0.02 & 2.95 & 1.80 & 0.065 \\
\hline & & & $\mathrm{C}_{3}-\mathrm{H}_{10}$ & $\sigma^{*}$ & & 0.69 & 1.34 & 0.027 \\
\hline \multirow[t]{6}{*}{$\mathrm{C}_{3}-\mathrm{C}_{4}$} & \multirow[t]{6}{*}{$\sigma$} & \multirow[t]{6}{*}{1.97} & $\mathrm{C}_{2}-\mathrm{C}_{3}$ & $\sigma^{*}$ & & 1.43 & 1.49 & 0.041 \\
\hline & & & $\mathrm{C}_{2}-\mathrm{H}_{9}$ & $\sigma^{*}$ & 0.007 & 1.89 & 1.72 & 0.051 \\
\hline & & & $\mathrm{C}_{3}-\mathrm{H}_{10}$ & $\sigma^{*}$ & & 3.58 & 1.71 & 0.070 \\
\hline & & & $C_{4}-C_{5}$ & $\sigma^{*}$ & 0.02 & 9.53 & 1.82 & 0.118 \\
\hline & & & $C_{4}-C_{7}$ & $\sigma^{*}$ & 0.01 & 5.98 & 1.75 & 0.092 \\
\hline & & & $\mathrm{C}_{5}-\mathrm{H}_{11}$ & $\sigma^{*}$ & 0.01 & 2.29 & 1.73 & 0.056 \\
\hline \multirow[t]{3}{*}{$\mathrm{C}_{3}-\mathrm{C}_{4}$} & \multirow[t]{3}{*}{$\pi$} & 1.97 & $C_{1}-C_{2}$ & $\pi^{*}$ & 0.23 & 21.75 & 1.02 & 0.134 \\
\hline & & & $\mathrm{C}_{1}-\mathrm{N}_{8}$ & $\sigma^{*}$ & 0.01 & 0.68 & 1.46 & 0.029 \\
\hline & & & $\mathrm{C}_{2}-\mathrm{C}_{3}$ & $\sigma^{*}$ & & 166.56 & 1.29 & 0.435 \\
\hline
\end{tabular}


PRABHA et al., Orient. J. Chem., Vol. 32(4), 1937-1945 (2016)

\begin{tabular}{|c|c|c|c|c|c|c|c|c|}
\hline & & & $\mathrm{C}_{4}-\mathrm{C}_{5}$ & $\sigma^{*}$ & 0.02 & 11.49 & 1.62 & 0.128 \\
\hline & & & $\mathrm{C}_{5}-\mathrm{N}_{6}$ & $\sigma^{*}$ & 0.03 & 0.84 & 1.43 & 0.032 \\
\hline & & & $\mathrm{C}_{5}-\mathrm{N}_{6}$ & $\pi^{*}$ & 0.73 & 13.14 & 0.80 & 0.107 \\
\hline & & & $\mathrm{C}_{5}-\mathrm{H}_{11}$ & $\sigma^{*}$ & 0.01 & 3.76 & 1.53 & 0.071 \\
\hline & & & $\mathrm{C}_{7}-\mathrm{H}_{13}$ & $\sigma^{*}$ & 0.007 & 2.54 & 1.47 & 0.058 \\
\hline & & & $\mathrm{C}_{7}-\mathrm{H}_{15}$ & $\sigma^{*}$ & 0.007 & 2.57 & 1.47 & 0.058 \\
\hline & & & $\mathrm{N}_{8}-\mathrm{H}_{17}$ & $\sigma^{*}$ & & 11.60 & 1.45 & 0.122 \\
\hline & & & $\mathrm{H}_{12}-\mathrm{O}_{27}$ & $\sigma^{*}$ & 0.063 & 2.55 & 3.45 & 0.08 \\
\hline $\mathrm{C}_{3}-\mathrm{H}_{10}$ & $\sigma$ & 1.97 & $C_{1}-C_{2}$ & $\sigma^{*}$ & & 3.67 & 1.45 & 0.065 \\
\hline & & & $\mathrm{C}_{2}-\mathrm{C}_{3}$ & $\sigma^{*}$ & & 4.83 & 1.09 & 0.065 \\
\hline & & & $\mathrm{C}_{2}-\mathrm{H}_{9}$ & $\sigma^{*}$ & 0.007 & 0.63 & 1.32 & 0.026 \\
\hline & & & $C_{3}-C_{4}$ & $\sigma^{*}$ & 0.02 & 5.23 & 1.77 & 0.086 \\
\hline & & & $C_{4}-C_{5}$ & $\sigma^{*}$ & 0.02 & 8.10 & 1.42 & 0.096 \\
\hline & & & $C_{4}-C_{7}$ & $\sigma^{*}$ & 0.01 & 0.68 & 1.35 & 0.027 \\
\hline$C_{4}-C_{5}$ & $\sigma$ & 1.97 & $\mathrm{C}_{3}-\mathrm{C}_{4}$ & $\sigma^{*}$ & 0.02 & 8.16 & 2.02 & 0.115 \\
\hline & & & $\mathrm{C}_{3}-\mathrm{H}_{10}$ & $\sigma^{*}$ & & 6.50 & 1.56 & 0.090 \\
\hline & & & $C_{4}-C_{7}$ & $\sigma^{*}$ & 0.01 & 2.08 & 1.59 & 0.052 \\
\hline & & & $C_{5}-N_{6}$ & $\sigma^{*}$ & 0.03 & 0.89 & 1.48 & 0.032 \\
\hline & & & $\mathrm{C}_{5}-\mathrm{H}_{11}$ & $\sigma^{*}$ & 0.01 & 1.41 & 1.57 & 0.042 \\
\hline & & & $\mathrm{N}_{6}-\mathrm{O}_{27}$ & $\sigma^{*}$ & 0.04 & 6.55 & 2.83 & 0.122 \\
\hline & & & $\mathrm{C}_{7}-\mathrm{H}_{14}$ & $\sigma^{*}$ & 0.002 & 0.68 & 1.52 & 0.029 \\
\hline$C_{4}-C_{7}$ & $\sigma$ & & $\mathrm{C}_{2}-\mathrm{C}_{3}$ & $\sigma^{*}$ & & 37.57 & 1.28 & 0.196 \\
\hline & & & $\mathrm{C}_{3}-\mathrm{C}_{4}$ & $\sigma^{*}$ & 0.02 & 6.86 & 1.96 & 0.103 \\
\hline & & & $\mathrm{C}_{3}-\mathrm{H}_{10}$ & $\sigma^{*}$ & & 0.82 & 1.50 & 0.031 \\
\hline & & & $C_{4}-C_{5}$ & $\sigma^{*}$ & 0.02 & 0.83 & 1.61 & 0.033 \\
\hline & & & $\mathrm{C}_{5}-\mathrm{N}_{6}$ & $\sigma^{*}$ & 0.03 & 7.77 & 1.42 & 0.094 \\
\hline & & & $\mathrm{C}_{5}-\mathrm{H}_{11}$ & $\sigma^{*}$ & 0.01 & 1.07 & 1.52 & 0.036 \\
\hline & & & $\mathrm{N}_{8}-\mathrm{H}_{17}$ & $\sigma^{*}$ & & 0.76 & 1.43 & 0.030 \\
\hline $\mathrm{C}_{5}-\mathrm{N}_{6}$ & $\sigma$ & 1.95 & $C_{1}-C_{2}$ & $\sigma^{*}$ & & 1.06 & 3.64 & 0.056 \\
\hline & & & $\mathrm{C}_{1}-\mathrm{N}_{6}$ & $\sigma^{*}$ & 0.08 & 9.60 & 3.37 & 0.162 \\
\hline & & & $\mathrm{C}_{2}-\mathrm{C}_{3}$ & $\sigma^{*}$ & & 251.96 & 3.28 & 0.816 \\
\hline & & & $\mathrm{C}_{4}-\mathrm{C}_{5}$ & $\sigma^{*}$ & 0.02 & 14.38 & 3.61 & 0.204 \\
\hline & & & $C_{4}-C_{7}$ & $\sigma^{*}$ & 0.01 & 1.12 & 3.54 & 0.057 \\
\hline & & & $\mathrm{C}_{5}-\mathrm{N}_{6}$ & $\sigma^{*}$ & 0.03 & 0.70 & 3.42 & 0.044 \\
\hline & & & $\mathrm{C}_{5}-\mathrm{H}_{11}$ & $\sigma^{*}$ & 0.01 & 6.17 & 3.52 & 0.133 \\
\hline & & & $\mathrm{N}_{6}-\mathrm{O}_{27}$ & $\sigma^{*}$ & 0.04 & 33.29 & 4.78 & 0.356 \\
\hline & & & $\mathrm{N}_{8}-\mathrm{H}_{17}$ & $\sigma^{*}$ & & 17.71 & 3.44 & 0.222 \\
\hline & & & $\mathrm{H}_{12}-\mathrm{O}_{27}$ & $\sigma^{*}$ & 0.06 & 10.94 & 5.44 & 0.219 \\
\hline$C_{5}-N_{6}$ & $\pi$ & 1.80 & $C_{1}-C_{2}$ & $\pi^{*}$ & 0.23 & 14.49 & 0.65 & 0.088 \\
\hline & & & $\mathrm{C}_{2}-\mathrm{C}_{3}$ & $\sigma^{*}$ & & 7.52 & 0.93 & 0.078 \\
\hline & & & $C_{3}-C_{4}$ & $\pi^{*}$ & 0.24 & 26.58 & 0.76 & 0.128 \\
\hline & & & $\mathrm{C}_{5}-\mathrm{N}_{6}$ & $\pi^{*}$ & 0.73 & 36.65 & 0.44 & 0.133 \\
\hline $\mathrm{C}_{5}-\mathrm{H}_{11}$ & $\sigma$ & & $C_{1}-N_{6}$ & $\sigma^{*}$ & & 3.33 & 1.20 & 0.057 \\
\hline & & & $\mathrm{C}_{3}-\mathrm{C}_{4}$ & $\sigma^{*}$ & 0.02 & 5.11 & 1.79 & 0.086 \\
\hline & & & $\mathrm{C}_{4}-\mathrm{C}_{5}$ & $\sigma^{*}$ & 0.02 & 1.91 & 1.44 & 0.047 \\
\hline & & & $\mathrm{C}_{5}-\mathrm{N}_{6}$ & $\sigma^{*}$ & 0.03 & 0.93 & 1.25 & 0.031 \\
\hline $\mathrm{N}_{6}-\mathrm{O}_{27}$ & $\sigma$ & 1.96 & $C_{1}-C_{2}$ & $\sigma^{*}$ & & 3.55 & 3.17 & 0.095 \\
\hline & & & $\mathrm{C}_{1}-\mathrm{N}_{6}$ & $\sigma^{*}$ & 0.08 & 10.68 & 2.91 & 0.160 \\
\hline & & & $\mathrm{C}_{2}-\mathrm{C}_{3}$ & $\sigma^{*}$ & & 0.60 & 2.82 & 0.037 \\
\hline & & & $\mathrm{C}_{5}-\mathrm{N}_{6}$ & $\sigma$ & 0.03 & 7.70 & 2.96 & 0.135 \\
\hline
\end{tabular}


PRABHA et al., Orient. J. Chem., Vol. 32(4), 1937-1945 (2016)

\begin{tabular}{|c|c|c|c|c|c|c|c|c|}
\hline & & & $\mathrm{N}_{6}-\mathrm{O}_{27}$ & $\sigma^{*}$ & 0.04 & 0.83 & 4.32 & 0.054 \\
\hline & & & $\mathrm{H}_{12}-\mathrm{O}_{27}$ & $\sigma^{*}$ & 0.06 & 73.96 & 4.98 & 0.546 \\
\hline \multirow{3}{*}{$\mathrm{C}_{7}-\mathrm{H}_{13}$} & \multirow[t]{3}{*}{$\sigma$} & \multirow[t]{3}{*}{1.98} & $\mathrm{C}_{2}-\mathrm{C}_{3}$ & $\sigma^{*}$ & & 4.11 & 1.08 & 0.060 \\
\hline & & & $C_{3}-C_{4}$ & $\sigma^{*}$ & 0.02 & 2.09 & 1.77 & 0.054 \\
\hline & & & $\mathrm{C}_{3}-\mathrm{C}_{4}$ & $\pi^{*}$ & 0.24 & 5.27 & 0.91 & 0.066 \\
\hline \multirow{3}{*}{$\begin{array}{l}\mathrm{C}_{7}-\mathrm{H}_{14} \\
\mathrm{C}_{7}-\mathrm{H}_{15}\end{array}$} & \multirow{3}{*}{$\begin{array}{l}\sigma \\
\sigma\end{array}$} & \multirow[t]{3}{*}{1.98} & $C_{4}-C_{5}$ & $\sigma^{*}$ & 0.02 & 7.26 & 1.42 & 0.091 \\
\hline & & & $\mathrm{C}_{3}-\mathrm{C}_{4}$ & $\sigma^{*}$ & 0.02 & 2.06 & 1.76 & 0.054 \\
\hline & & & $C_{3}-C_{4}$ & $\pi^{*}$ & 0.24 & 5.27 & 0.91 & 0.066 \\
\hline \multirow[t]{2}{*}{$\mathrm{N}_{8}-\mathrm{H}_{16}$} & \multirow[t]{2}{*}{$\sigma$} & \multirow[t]{2}{*}{1.98} & $C_{1}-C_{2}$ & $\sigma^{*}$ & & 1.74 & 1.61 & 0.047 \\
\hline & & & $C_{1}-C_{2}$ & $\pi^{*}$ & 0.23 & 2.38 & 0.99 & 0.046 \\
\hline \multirow{2}{*}{$\mathrm{N}_{8}-\mathrm{H}_{17}$} & \multirow[t]{2}{*}{$\sigma$} & \multirow[t]{2}{*}{1.98} & $C_{1}-C_{2}$ & $\pi^{*}$ & 0.23 & 2.17 & 0.98 & 0.044 \\
\hline & & & $\mathrm{C}_{1}-\mathrm{N}_{6}$ & $\sigma^{*}$ & 0.08 & 5.09 & 1.34 & 0.075 \\
\hline \multirow[t]{5}{*}{$\mathrm{H}_{12}-\mathrm{O}_{27}$} & \multirow[t]{5}{*}{$\sigma$} & \multirow[t]{5}{*}{1.85} & $\mathrm{C}_{1}-\mathrm{N}_{6}$ & $\sigma^{*}$ & 0.08 & 22.45 & 2.41 & 0.211 \\
\hline & & & $\mathrm{C}_{2}-\mathrm{C}_{3}$ & $\sigma^{*}$ & & 17.06 & 2.31 & 0.183 \\
\hline & & & $\mathrm{C}_{5}-\mathrm{N}_{6}$ & $\pi^{*}$ & 0.73 & 0.76 & 1.83 & 0.040 \\
\hline & & & $\mathrm{N}_{6}-\mathrm{O}_{27}$ & $\sigma^{*}$ & 0.04 & 15.62 & 3.81 & 0.224 \\
\hline & & & $\mathrm{N}_{8}-\mathrm{H}_{17}$ & $\sigma^{*}$ & & 1.31 & 2.47 & 0.053 \\
\hline \multirow{3}{*}{$C_{1}-C_{2}$} & \multirow[t]{3}{*}{$\pi^{*}$} & \multirow[t]{3}{*}{0.23} & $\mathrm{C}_{3}-\mathrm{C}_{4}$ & $\pi^{*}$ & 0.24 & 37.04 & 0.10 & 0.111 \\
\hline & & & $\mathrm{N}_{8}-\mathrm{H}_{16}$ & $\sigma^{*}$ & 0.01 & 0.78 & 0.45 & 0.047 \\
\hline & & & $\mathrm{N}_{8}-\mathrm{H}_{17}$ & $\sigma^{*}$ & & 1.07 & 0.43 & 0.055 \\
\hline \multirow[t]{2}{*}{$\mathrm{C}_{3}-\mathrm{C}_{4}$} & \multirow[t]{2}{*}{$\pi^{*}$} & \multirow[t]{2}{*}{0.24} & $\mathrm{C}_{7}-\mathrm{H}_{13}$ & $\sigma^{*}$ & 0.007 & 1.38 & 0.35 & 0.056 \\
\hline & & & $\mathrm{C}_{7}-\mathrm{H}_{15}$ & $\sigma^{*}$ & & 1.37 & 0.35 & 0.055 \\
\hline \multirow{2}{*}{$C_{5}-N_{6}$} & \multirow[t]{2}{*}{$\pi^{*}$} & \multirow[t]{2}{*}{0.73} & $C_{1}-C_{2}$ & $\pi^{*}$ & 0.23 & 16.49 & 0.22 & 0.077 \\
\hline & & & $C_{3}-C_{4}$ & $\pi^{*}$ & 0.24 & 31.75 & 0.32 & 0.128 \\
\hline
\end{tabular}

describes the bonding antibonding interaction quantitatively and is expressed by means of secondorder perturbation interaction energy $E_{(2)}$ and also provides a convenient basis for investigating charge transfer or conjugative interaction in molecular systems. Some electron donor orbital, acceptor orbital and the interacting stabilization energy resulting from the second order micro disturbance theory are reported. NBO analysis has been performed on

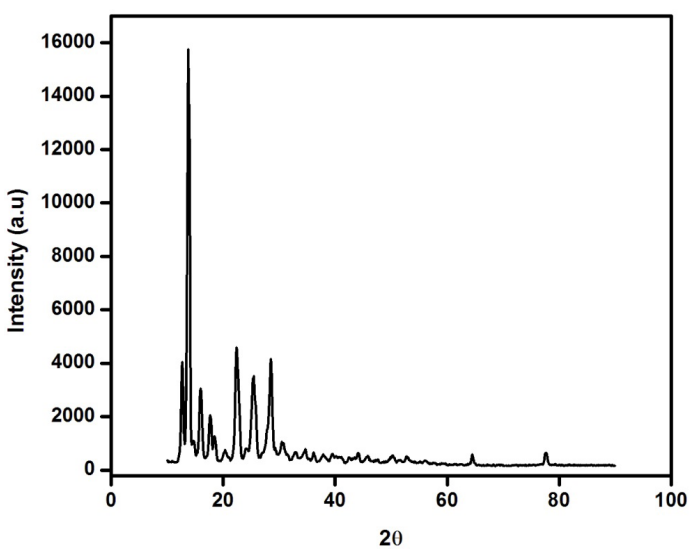

Fig. 5: Powder XRD pattern of 2A5MPSA the molecule at a B3LYP/6-311++G level in order to elucidate the intramolecular, rehybridization and delocalization of electron density within the molecule, which is presented in Table 4. The result of the interaction is a loss of occupancy from the concentration of electron NBO of the idealized Lewis structure into an empty non-Lewis orbital. For each donor (i) and acceptor (j), the stabilization energy $E_{(2)}$ associated with the delocalization $i \rightarrow j$ is estimated as

Where

$$
\begin{aligned}
\mathrm{E}_{(2)} & =-\mathrm{n}_{\sigma}<\sigma|\mathrm{F}| \sigma>^{2} / \varepsilon_{\sigma}{ }^{*}-\varepsilon_{\sigma} \\
& =-\mathrm{n}_{\sigma} \mathrm{F}_{\mathrm{ij}}{ }^{2} / \Delta \mathrm{E}
\end{aligned}
$$

$<\sigma|\mathrm{F}| \sigma\rangle^{2}$ or $\mathrm{F}_{\mathrm{ij}}^{2}$ is the Fock matrix element I and j NBO orbital's, $\varepsilon_{\sigma}{ }^{*}$ and $\varepsilon_{\sigma}$ are the energies of ó and $\sigma^{*}$ NBO's and $n_{\sigma}$ is the population of the donor $\sigma$ orbital. The intramolecular interactions are formed by the orbital overlap between bonding (C-C) and (C-C) anti-bond orbital which results from intramolecular charge transfer (ICT) causing stabilization of the orbital that weakens the respective bonds. The strong intramolecular hyper conjugative interactions of the $\sigma$ electron of $\left(\mathrm{C}_{1}-\mathrm{C}_{2}\right)$ distribute to 
$\sigma^{*} \mathrm{C}_{1}-\mathrm{N}_{8} \mathrm{C}_{2}-\mathrm{H}_{9}$ and $\mathrm{N}_{8}-\mathrm{H}_{16}$ of the ring. The $\pi\left(\mathrm{C}_{3}-\mathrm{C}_{4}\right)$ bond is interacting with $\pi^{\star}\left(\mathrm{C}_{1}-\mathrm{C}_{2}\right)$ with the energy $21.75 \mathrm{kcal} / \mathrm{mol}$ for 2A5MPSA.The $\sigma\left(\mathrm{C}_{1}-\mathrm{N}_{6}\right)$ bond is contributing energy by $0.55 \mathrm{kcal} / \mathrm{mol}$ with $\sigma^{*}\left(\mathrm{C}_{2}-\mathrm{C}_{3}\right)$. In the reverse trend, the $\sigma\left(\mathrm{C}_{1}-\mathrm{N}_{8}\right)$ bond is contributing energy by $2.53 \mathrm{kcal} / \mathrm{mol}$ with $\sigma^{\star}\left(\mathrm{C}_{1}-\mathrm{C}_{2}\right)$.

\section{CONCLUSION}

Organic single crystal of 2-amino 5-methyl pyridinium salicylate (2A5MPSA) crystal was grown by slow evaporation method at room temperature. The grown crystals were characterized by powder XRD analysis. The powder XRD pattern confirms the crystalline nature of the crystal. The presence of various functional groups present in the grown crystal was confirmed by the FTIR analysis. The good transparency in the entire visible region and low cut-off wavelength facilitate the 2A5MPSA crystal to be a potential material for NLO applications.

The TGA/DTA analysis revealed that the grown 2A5MPSA crystal can be exploited for NLO applications up to $170^{\circ} \mathrm{C}$. The NBO analysis revealed that inter and intra molecular interactions in the molecular system of 2A5MPSA. Mulliken charge analysis performed using the optimized geometry of the molecule provided vital details on the charge distribution 2A5MPSA. The NLO property was confirmed by first order hyperpolarizability values of 2A5MPSA. Further, HOMO-LUMO energy values support the NLO activity of 2A5MPSA. Thus the all above results confirm that the 2A5MPSA crystal is suitable for biological and optoelectronic device applications.

\section{REFERENCES}

1. Mariappan, L.; Kandasamy, A.; RathnaKumari, M.; Sureshkumar, P.; Optik, 2013, 124, 26302634.

2. Kandasamy, A.; Siddeswaran, R.; Murugakoothan, P.; Sureshkumar, P.; Mohan, R.; Cryst Growth Des. 2007, 7, 183-186.

3. Prasad, P.N.; Williams D.J, Wiley 1991.

4. Bella, S.D.; Fragala, I.; Guerri, A.; Dapporto, P.; Nakatani, K.; Inorg. Chem. Acta, 2004, 357, 19.

5. Sieman (Ed) A.E, Springer, 1991, 64.

6. Meir, U.; Bosch, M.; Boshard, C.; Gunter P.; Synth. Met, 2000, 109, 19-22.

7. Siddhewaran, R.; Sankar, R.; Rathnakumari, M.; Murugakoothan, P.; Jayavel, R.; Suresh kumar, P.; Surf. Rev. Lett. 2006, 13, 803-
808.

8. Mohammad a, Halim.; Dawn M. Shaw.; Raymond A. Poirer, J. Mol. Struc. (Theo Chem), 2010, 960, 63-72.

9. Thanthiriwatte, K .S.; De Silva KMN.; J. Mol. Struc. (Theo Chem), 2002, 617, 169-175.

10. Bartkowaik, W.; Misiaszek, T.; Chem. Phys. 2000, 261, 353-357.

11. Chaitanya, K.; Spectrochim. Acta Part A 2012 , 86,159-173.

12. Fatimah Ali Al-Zahrani, Muhammad Nadeem Arshad, Abdullah M. Asiri, Tariq Mahmood, Mazhar Amjad Gilani and Reda M. Elshishtawy, Chem. Central J. 2016, 10-13.

13. Venkatesh, G.; Govindaraju, Vennila, P.; Ind. J. Chem. 2016, 55A, 413-422. 